\title{
Efficacy of Panobinostat for the Treatment of Multiple Myeloma
}

\section{Evangelos Eleutherakis-Papaiakovou (D), Nikolaos Kanellias, Efstathios Kastritis, Maria Gavriatopoulou, Evangelos Terpos $\mathbb{D}^{D}$, and Meletios Athanasios Dimopoulos}

Department of Clinical Therapeutics, National and Kapodistrian University of Athens, School of Medicine, Alexandra General Hospital, Athens, Greece

Correspondence should be addressed to Evangelos Eleutherakis-Papaiakovou; mdeleutherakis2@gmail.com

Received 9 November 2019; Accepted 27 December 2019; Published 13 January 2020

Academic Editor: Thomas R. Chauncey

Copyright (C) 2020 Evangelos Eleutherakis-Papaiakovou et al. This is an open access article distributed under the Creative Commons Attribution License, which permits unrestricted use, distribution, and reproduction in any medium, provided the original work is properly cited.

\begin{abstract}
Panobinostat represents a potent oral nonselective pan-histone deacetylase inhibitor (HDAC) with activity in myeloma patients. It has been approved by the FDA and EMA in combination with bortezomib and dexamethasone for the treatment of multiple myeloma, in patients who have received at least two prior regimens, including bortezomib and an immunomodulatory agent. In order to further explore its clinical potential, it is evaluated in different combinations in relapsed/refractory and newly diagnosed multiple myeloma. This review focuses on available data about panobinostat's pharmacology and its role in clinical practice. This review will reveal panobinostat's efficacy as antimyeloma treatment, describing drug evolution from preclinical experimental administration to administration in phase III trials, which established its role in current clinical practice. Based on the latest data, we will present its mechanism of action, its efficacy, and most important issues regarding its toxicity profile. We will further try to shed light on its role in current and future therapeutic landscape of myeloma patients. Panobinostat retains its role in therapy of multiple myeloma because of its manageable toxicity profile and its efficacy, mainly in heavily pretreated multiple myeloma patients. These characteristics make it valuable also for novel regimens in combination with second-generation proteasome inhibitors, IMiDs, and monoclonal antibodies. Results of ongoing trials are expected to shed light on drug introduction in different therapeutic combinations or even at an earlier level of disease course.
\end{abstract}

\section{Introduction}

Multiple myeloma is a plasma cell dyscrasia characterized by clonal plasma cell proliferation within bone marrow and increased production of monoclonal paraprotein, excreted in the blood or urine. It mainly affects elderly population, with a median age of diagnosis at approximately 70 years [1]. It is the third most common hematopoietic malignancy (after lymphoma and leukemia), representing approximately $13 \%$ of hematologic malignancies and $1 \%$ of all cancers $[2,3]$. In 2018 , it was estimated that 30,770 patients in the USA would be diagnosed with multiple myeloma and 12,770 patients will succumb to myeloma disease [4]. Globally, it is estimated that in 2018, 159,985 patients will be diagnosed with multiple myeloma and 106,105 patients will expire due to myeloma disease [5]. Due to continuous population aging, the incidence of myeloma is expected to rise in time. Typical clinical disease manifestations include anemia, hypercalcemia, renal insufficiency, and myeloma bone disease, known also as the "CRAB" features. Despite advances in disease's early detection, including recently introduced biological markers (abnormal FLC ratio, bone marrow infiltration by clonal plasma cells $>60 \%$, and more than one focal lesion in $\mathrm{MRI})$, the aforementioned $\mathrm{CRAB}$ features remain the hallmark of active multiple myeloma disease [6].

Initial therapeutic management of multiple myeloma with conventional chemotherapy attained poor results $[7,8]$. The introduction of novel agents [9], such as proteasome inhibitors and immunomodulatory drugs [10-13], and incorporation of autologous stem cell transplantation in clinical practice [14-16] has significantly reformed therapeutic landscape of multiple myeloma patients and vastly increased their outcome, by improving significantly the response rate and depth of response. Superior therapeutic 
efficacy of novel agents has been translated into prolonged progression-free survival (PFS) and overall survival (OS). Recent introduction of second-generation novel agents (such as carfilzomib [17] and pomalidomide [18, 19]) and monoclonal antibodies (such as daratumumab [20-24], isatuximab [25-28], and elotuzumab [29-31]) in multiple myeloma therapeutic setting has rapidly evolved therapeutic management, especially for refractory/relapsed multiple myeloma patients. Before the introduction of more advanced novel agents (carfilzomib and pomalidomide), patients with relapsed/refractory myeloma after initial therapy with proteasome inhibitors and IMiDs attained a dismal prognosis, with a median PFS of 5 months and a median OS not exceeding 9 months [32].

Despite major therapeutic advances in multiple myeloma therapy, it remains an incurable disease. Initial response to the aforementioned therapeutic agents is usually transient. Due to the evolvement of multiple malignant clones, multiple myeloma patients finally relapse, with the emergence of a more resistant myeloma cell population, requiring new lines of treatment. Most patients receive multiple lines of therapy during the course of their disease [33]. However, after each relapse, duration of subsequent response usually shortens, revealing an unmet medical need for effective therapies for heavily pretreated patients [34, 35]. The aforementioned data underline the importance of continuous research for agents with new mechanisms of action that will continue to offer a clinical benefit in multiple myeloma patients refractory/relapsed to current therapeutic regimens. Ideally, agents should be active through novel mechanisms of action and should be effective as monotherapy with panobinostat should resensitize patients to previously administered therapeutic agents.

Panobinostat (chemical name: 2-hydroxypropanoic acid, compound with 2-(E)-N-hydroxy-3-[4-[[[2-(2-methyl1H-indol-3- yl)ethyl]amino]methyl]phenyl]-2-propenamide $[1: 1]$, trademark Farydak) is a first-in-class potent pan-deacetylase (DAC) inhibitor [36] that has been approved in February 2015 by the US FDA (US Food and Drug Administration) in combination with bortezomib and dexamethasone for the treatment of multiple myeloma, in patients who have received at least two prior regimens, including bortezomib and an immunomodulatory agent $[37,38]$. The EMA (European Medicines Agency) has also granted approval in August 2015 for this agent [39], which is also recommended by the NICE (National Institute for Health and Care Excellence) [40] for the same indication.

\section{HDAC System}

DAC enzymes, also known as histone deacetylases (HDACs) are proteins that mainly act on histone proteins and transcription factors. Histones are responsible for DNA arrangement into nucleosomes. Histone modifications by histone deacetylases lead to different chromatin conformation, permitting DNA transcription [41]. Therefore, histone deacetylases and histone acetyltransferases by their opposing activities regulate gene expression, cell differentiation, and survival [42-45]. Some members of the HDAC family are transferred from the nucleus to cytoplasm and mainly act on nonhistone proteins. These proteins are categorized into four main classes (I, II, III, and IV). Preclinical studies with myeloma cells have shown that tumor suppressor genes are silenced in these cells $[46,47]$. Treatment of myeloma cell lines with HDAC inhibitors like panobinostat induces reactivation of suppressed genes, leading eventually to cell death. In further studies with myeloma cells, DAC inhibition resulted in upregulation of genes that have been related to increased overall survival (OS) [48]. These preclinical trials demonstrated that pharmaceutical manipulation of HDACs had antimyeloma activity and should be further accessed in clinical trials. In fact, several DAC agents have been already evaluated in clinical trials like panobinostat, vorinostat [49-51], ricolinostat [52, 53], romidepsin [54], and ACY-241 [55]. Among HDAC inhibitors, panobinostat remains the only approved agent for the treatment of refractory/relapsed multiple myeloma patients.

\section{Drug Chemistry}

Panobinostat (LBH-589) is an orally administered hydroxamic acid pan-DAC inhibitor (Table 1) [36]. It has shown preclinical activity in all four classes of HDACs. At low concentrations, it induces apoptosis in multiple myeloma cells resistant to conventional therapies. Moreover, it inhibits cell proliferation even in the presence of BMSCs and it induces caspase activation and poly-(ADP-ribose) polymerase (PARP) cleavage in myeloma cell lines. It is worth to mention that deacetylase HDAC6 seems to regulate aggresome formation [56], an alternative cleavage pathway for catabolism of misfolded proteins that develop when proteasomes are incapable of cleaving misfolded ubiquitinated proteins. Therefore, proteasome inhibitors such as bortezomib, by inhibiting proteasome cleavage mechanism, induce aggresome formation due to overload of excess proteins while panobinostat through inhibition of HDAC6 blocks aggresome formation [57]. Their combination in myeloma cells lines results in synergistic toxicity against myeloma due to the complementary blocking of these complementary protein degradation pathways, the aggresome and the proteasome, leading to increased accumulation of excess proteins, and eventually signals the cell to undergo apoptosis. Bortezomib also targets and downregulates class I HDACs, indicating a different mechanism of HDAC inhibition, complementary to that of HDAC inhibitors [58-60]. These preclinical observations provided the rational for subsequent clinical trials combining panobinostat with bortezomib.

\section{Pharmacokinetics and Metabolism}

After receiving orally a dose of $20 \mathrm{mg}$ panobinostat, it is rapidly absorbed, achieving maximum plasma concentration within 0.5 to 2 hours $[61,62]$. When orally administered, the median maximum plasma concentration of panobinostat was $5.5-21.2 \mathrm{ng} / \mathrm{ml}$. The absolute bioability of orally administered panobinostat was $21 \%$. Drug bioability and rate of absorption was not significantly affected by food 
TABLE 1: Drug summary.

\begin{tabular}{|c|c|}
\hline Generic drug name & Panobinostat \\
\hline Market name (pharmaceutical company) & Farydac $^{\circledR}$ (Novartis) \\
\hline Phase & Approved \\
\hline Indication & $\begin{array}{c}\text { In combination with bortezomib and } \\
\text { dexamethasone, for the treatment of adult patients } \\
\text { with relapsed and/or refractory multiple myeloma } \\
\text { who have received at least two prior regimens } \\
\text { including bortezomib and an immunomodulatory } \\
\text { agent }\end{array}$ \\
\hline Pharmacology description/mechanism of action & HDAC inhibitor \\
\hline Route of administration & Oral \\
\hline Chemical structure & $\begin{array}{l}\text { 2-Hydroxypropanoic acid, compound with } 2-(\mathrm{E})-\mathrm{N} \text { - } \\
\text { hydroxy-3-[4-[[[2-(2-methyl-1H-indol-3-yl)ethyl] } \\
\text { amino]methyl]phenyl]-2-propenamide }[1: 1]\end{array}$ \\
\hline Pivotal trial $(\mathrm{s})$ & Panorama 1 \\
\hline Metabolism and elimination & $\begin{array}{l}\text { Extensively metabolized, eliminated equally through } \\
\text { the kidney and liver }\end{array}$ \\
\hline Main toxicities & $\begin{array}{l}\text { Thrombocytopenia, anemia, neutropenia, diarrhea, } \\
\text { fatigue }\end{array}$ \\
\hline
\end{tabular}

intake. Panobinostat has a half-life of 31 hours and is excreted in feces and urine, nearly equally among all patients $[63,64]$. Panobinostat is metabolized via hydrolysis, acidosis, and glucuronidation through mainly noncytochrome P450 (CYP) enzymes and CYP enzymes. CYP3A4 inhibitors may significantly increase panobinostat exposure, thus requiring close monitoring for drug interactions or even panobinostat dose reduction. On the contrary, CYP34 inducers may significantly reduce panobinostat exposure and should be avoided when possible.

Panobinostat may also inhibit CYPY2D6 and relevant substrates should be avoided when possible. Panobinostat administration has been reported to induce QT prolongation, especially in intravenous administered form. Therefore, concomitant administration of agents that prolong QT should be avoided. Regarding panobinostat administration and renal or hematological impairment, earlier studies have shown that renal function did not affect drug clearance and tolerability while hepatic impairment was associated with reduced drug clearance and increased plasma exposure $[65,66]$.

\section{Administration Schedule}

According to manufacturer's labeling, panobinostat is orally administered in a schedule of $20 \mathrm{mg}$ starting dose once every other day for 3 doses each week during weeks 1 and 2 of a 21 day cycle for up to 8 cycles [67]. The total duration of therapy may continue up to 163 -week cycles if the patient shows clinical benefit and manageable toxicity. After eight cycles of treatment, bortezomib administration is modified while panobinostat dosing remains unchanged. Renal impairment does not require dose modification. In mild hepatic impairment, due to increased drug exposure, it is recommended to reduce panobinostat starting dose. Panobinostat administration is contradicted in severe hepatic impairment.
If dose reduction is required, administration may be reduced at $5 \mathrm{mg}$ intervals. Panobinostat should be discontinued if a dose of $10 \mathrm{mg}$ three times a week is not tolerable.

\section{Clinical Trials of Panobinostat in Multiple Myeloma Patients}

6.1. Panobinostat as Monotherapy. Encouraging results from preclinical trials led to evaluation of monotherapy with panobinostat in clinical studies. In a pioneer phase Ia/II trial with various hematologic malignancies, maximum tolerated dose has been determined at $60 \mathrm{mg}$ weekly [68]. In this trial, 12 patients with multiple myeloma received panobinostat and only one of them achieved PR (partial response), indicating a poor result as monotherapy. Panobinostat was further evaluated as monotherapy in a clinical trial with patients suffering from refractory/relapsed multiple myeloma. In a phase II trial, 38 heavily pretreated multiple myeloma patients (with a median of five prior therapies) received $20 \mathrm{mg}$ panobinostat three times weekly until disease progression or intolerance [69]. Monotherapy with panobinostat resulted in also limited clinical activity with $2 / 38$ patients achieving partial response and minor response (MR), respectively, which were durable. Furthermore, 9/38 patients achieved stable disease (SD).

6.2. Panobinostat with Bortezomib and Dexamethasone. Initial preclinical studies with myeloma cell lines indicating synergistic activity of panobinostat and bortezomib and poor results from clinical trials evaluating panobinostat monotherapy provided the rationale for clinical evaluation of combinations of panobinostat with bortezomib and dexamethasone. In a phase Ib trial establishing maximum tolerated doses (MTD) for panobinostat, bortezomib, and dexamethasone in refractory or relapsed and refractory 
multiple myeloma patients [70], 52.9\% of patients achieved at least PR. It is worth to mention that among bortezomib refractory patients, $26.3 \%$ achieved at least PR, after reexposure to bortezomib with panobinostat, indicating that addition of panobinostat restored sensitiveness in a subgroup of previously refractory patients. These encouraging results led to further exploration of panobinostat's safety and effectiveness in a phase II trial (Panorama 2) [71] and in a phase 3 trial (Panorama 1) [72], in refractory/relapsed multiple myeloma patients.

Panorama 2 clinical trial included 55 patients. These patients had received at least 2 lines of treatment (with a median number of four previous lines of therapy, including an immunomodulatory drug) and were relapsed/refractory to bortezomib (progression within 60 days from the last dose of bortezomib). $34.5 \%$ of patients achieved at least PR with a median progression-free survival (PFS) of 5.4 months. This trial demonstrated the clinical benefit from panobinostat administration providing the rational for conducting a large multicenter randomized phase III trial, Panorama 1 trial. In this pivotal trial, 768 patients were randomly assigned in a $1: 1$ ratio to receive therapy with panobinostat-bortezomib-dexamethasone or placebo-bortezomib-dexamethasone. Patients had received 1 to 3 prior lines of treatment. Primary refractory myeloma patients and bortezomib refractory myeloma patients were excluded. The primary endpoint of this trial was PFS and was met as patients in the panobinostat arm achieved a median PFS of 12 months, compared to 8.1 months in the placebo arm $(p<0.0001)$. Median duration of response was also higher in the panobinostat arm (12 months vs. 7 months, respectively). Despite that, response rates were similar in both groups, patients receiving panobinostat achieved more frequently deeper than PR response (VGPR/CR) (28\% vs. $16 \%$ compared to patients that received placebo ( $p=0.00006)$. There was a trend towards superior survival for patients that received panobinostat that was sustained in the final overall survival analysis of this trial but did not reach statistical significance [73]. Based on these favorable results, the FDA granted approval in February 2015 for panobinostat combined with bortezomib and dexamethasone for relapsed and refractory multiple myeloma patients who have received at least 2 prior lines of treatment, including bortezomib and an IMiD.

Panobinostat has been further evaluated in combination with bortezomib and dexamethasone as induction therapy in multiple myeloma patients eligible for autologous stem cell transplantation with high-dose melphalan as conditioning regimen [74]. Compared to clinically established bortezomib-based induction regimens (VRD, VCD, VTD), this combination attained lower response rate, lower quality of response at a cost of significant discontinuation rate, and frequent drug dose reduction, rendering it rather unsuitable as pretransplant regimen.

Noteworthy, in the aforementioned trials, bortezomib was administered mainly intravenously. In the PANEX clinical trial [75], 87\% (34 of 39) of patients received subcutaneously bortezomib with panobinostat and dexamethasone. All patients were heavily pretreated with a median of four previously administered treatment regimens. In the subcutaneously administered bortezomib population, $62 \%$ of patients achieved at least PR and 15\% of patients achieved minimal response. The combination demonstrated a marked clinical benefit with concerns about regimen tolerability, since $28 \%$ of patients discontinued therapy due to adverse events. Nonetheless, it is worth to notice the short duration of therapy (8.1 weeks), mainly due to progressive disease, as probably expected in this heavily pretreated group of patients.

6.3. Panobinostat and Carfilzomib. Synergistic activity of panobinostat and the first-in-class proteasome inhibitor bortezomib provided the rational for exploring potential synergy of panobinostat with more advanced proteasome inhibitors like carfilzomib. Preclinical data in human multiple myeloma cell lines where panobinostat and carfilzomib were combined indicated a synergistic inhibition of myeloma cell survival, by inducing apoptosis, mitochondrial injury, and caspase activation. Oxidative damage through generation of ROS (reactive oxygen species) and inhibition of ERK1/2 pathway that normally protects myeloma cells from apoptosis were considered to be the main mechanisms of carfilzomib-panobinostat-induced myeloma cells' apoptosis [76]. Based on initial encouraging preclinical data, a phase I/II study of carfilzomib/panobinostat in refractory/ relapsed multiple myeloma patients has been conducted [77]. In this clinical trial, 44 heavily pretreated patients (median number of five prior regimens) were treated with this combination. Among them, $67 \%$ achieved at least PR irrespective of prior treatments. Adverse cytogenetics did not appear to affect response rate. The median time to progression (TTP) was 7.7 months while better quality of response predicted longer progression-free survival. After a median follow-up of 17 months, the overall survival has not been reached rendering this combination an effective regimen in these heavily pretreated patients. Combination of panobinostat with carfilzomib was further explored in another phase I trial [78]. 32 patients with a median number of 4 previously administered therapies received a median of 8 treatment cycles with carfilzomib and panobinostat. 57\% of patients achieved at least PR with a median PFS of 8 months and a median OS of 23 months. Noteworthy, in both previously mentioned clinical trials, steroids were not administered, without affecting the efficacy and safety of these steroid-sparing regimens, which were equally effective in bortezomib-sensitive and bortezomib-refractory patients.

6.4. Panobinostat and Lenalidomide. Combination of lenalidomide and panobinostat was evaluated in a phase 2 clinical trial [79]. 27 heavily pretreated patients with a median of 3 prior lines of treatment received this combination. Among them, 17 (63\%) showed high-risk cytogenetics. $41 \%$ of patients achieved at least PR, a remarkable result considering that $81 \%$ of patients were initially lenalidomide refractory. The median PFS was 7.1 months while in the lenalidomide refractory group, the median PFS was 6.5 months.

6.5. Panobinostat and Other Combinations. In vivo and in vitro preclinical data demonstrated synergistic activity of 
panobinostat with chemotherapeutic agents [80] like melphalan and anthracyclines [81]. Based on the aforementioned important data, a phase 2 study was designed, administering panobinostat combined with melphalan, thalidomide, and dexamethasone in relapsed/refractory multiple myeloma patients. Patients received 6 cycles of MPT-panobinostat and maintenance therapy with panobinostat plus prednisone. Due to severe drug-induced toxicity, dosage of panobinostat was reduced to $10 \mathrm{mg}$ three times weekly. At least $45 \%$ of patients achieved at least PR with a median PFS at 8.1 months and a median OS at 18.2 months, at cost of significant toxicity [82].

Positive results from clinical trials combining bortezomib or thalidomide with panobinostat encouraged the evaluation of a VTD-panobinostat regimen for relapsed/ refractory multiple myeloma patients [83]. A phase I/II open-label trial was conducted including 57 patients with refractory or relapsed multiple myeloma that had received a median of 1 line of treatment. Patients could receive 6 cycles of intense therapy and continue with panobinostat monotherapy as maintenance. 46 patients received the recommended dose of $20 \mathrm{mg}$ for panobinostat. Among them, 91\% achieved at least PR, with a median PFS of 16.1 months, and median OS was not reached, after a median follow-up of 28 months. Treatment was well tolerated. Most adverse events were mild or moderate with few cases of grade 3-4 diarrhea or fatigue, partially attributed to subcutaneous administration of bortezomib. In this trial, the VTD-panobinostat regimen emerged as an efficient and safe therapeutic modality. However, it must be underlined that $80 \%$ of patients had received only one previous line of treatment and patient refractory to bortezomib were excluded, leading to a highly selected drug-sensitive trial population. Favorable results in the first relapse prompted evaluation of panobinostat with VRD in a pretransplant phase I clinical trial, in order to potentially increase response rate and quality of response and reduce tumor burden before stem cell collection [84]. All 55 treated patients received at least one cycle of therapy. 95\% of them achieved at least PR, and $22 \%$ of them achieved at least $\mathrm{CR}$, a $\mathrm{CR}$ rate rather equal to previously reported for VRD regimen. Noteworthy, all patients who underwent stem cell collection after 4 cycles of VRD-panobinostat managed to collect a sufficient number of stem cells. VRDpanobinostat regimen has also been evaluated in refractory/ relapsed multiple myeloma patients. 16 patients that had already received a median of four prior lines of therapy received this regimen achieving 5 months of PFS and 13 months of overall survival [85]. Finally, panobinostat has been evaluated in even more heavily pretreated patients [86], refractory after $>10$ previous lines of treatment, combined with carfilzomib, thalidomide, cyclophosphamide, and dexamethasone, offering disease control with manageable toxicity, justifying further evaluation of panobinostat-based combinational regimens.

6.6. Panobinostat and Immunotherapy. There are few available data regarding combination of panobinostat and immunotherapy in multiple myeloma. Enhanced antimyeloma activity of daratumumab and panobinostat combination has been demonstrated in preclinical models [87]. Panobinostat has been shown to increase CD38 expression on myeloma cells from patients with newly diagnosed multiple myeloma or refractory/relapsed disease. Increased CD38 expression has been associated with a higher response rate in daratumumab monotherapy, probably due to increased daratumumab-associated ADCC [88]. In order to exploit a possible synergistic activity of these agents, myeloma cell lines were subsequently exposed to panobinostat and daratumumab, eliminating much more myeloma cells compared to daratumumab monotherapy. Clinical trials are warranted to confirm if this combination may offer additional clinical benefit by increasing response rate and extending duration of response, compared to daratumumab monotherapy.

PD-1/PD-L1 axis has also been evaluated in myeloma cell lines with conflicting results. Immune checkpoint inhibitors have been evaluated in multiple myeloma with lenalidomide or pomalidomide with modest results, mainly due to increased toxicity, high risk of mortality, and uncontrolled immunoreactivity [89-92]. Ongoing trials are now exploring the combination of anti-PD-1/PD-L1 agents with anti-CD38 monoclonal antibodies. Combinations of HDAC inhibitors with anti-PD-L1 agents have been shown to synergistically reduce melanoma cell survival, due to HDAC inhibitors' ability to induce prolonged PD-L1 expression in both human and mouse melanoma cell lines $[93,94]$. These combinations have also been evaluated in clinical trials (NCT02935790 and NCT02032810) in patients with advanced melanoma. Regarding multiple myeloma, preclinical studies have evaluated the combination of an anti-PD-L1 antibody with panobinostat [95] or other HDAC inhibitors, showing enhanced cytotoxicity [96]. These results should also be evaluated in clinical trials in order to confirm if restored immune function and enhanced cytotoxicity from these combinations may be translated in additional clinical benefit.

\section{Safety Issues and Strategies of Management of Key Adverse Events}

Panobinostat toxicities have been reported to be primarily of gastrointestinal and hematologic origin. Its toxicity profile shares strong resemblance to that of other HDAC inhibitors, including vorinostat and romidepsin. Initial trials with panobinostat monotherapy revealed main issues of drug's toxicity profile. Panorama I clinical trial provided a vast amount of data regarding panobinostat toxicity profile and its effective management. Most common severe adverse events (grade 3/4) were hematologic, including thrombocytopenia, anemia, and neutropenia [97, 98]. Gastrointestinal disorders and their management remain an important clinical issue for this group of patients. Diarrhea is the most common nonhematologic adverse event, affecting $76 \%$ of patients (33\% of grade $3 / 4)$, followed by fatigue $(60 \%, 26 \%$ of grade $3 / 4$ ). Drug toxicities necessitated dose modification or even drug interruption in the vast majority of patients [72, 99-102]. Despite drug toxicities, the proportion of on 
treatment deaths was similar between panobinostat and placebo arm. Moreover, deaths due to progressive disease were reported to be being slightly higher in the placebo arm. Noteworthy, administration of early intravenous formulations of panobinostat showed the potential to prolong QT interval in some patients [103]. In subsequent clinical trials with the oral form, QT prolongation frequency was equal to placebo arm [104]. However, QT prolongation is a dose dependent drug-associated toxicity and can appear more frequently with increased doses of oral panobinostat. Moreover, arrhythmias and cardiac-related deaths were more frequently reported during panobinostat administration [71]. Therefore, patients should have an ECG with normal QTc interval before initiating therapy with panobinostat and have monitoring with repeated ECGs of QTc while on therapy. Concomitant medications known to prolong QT interval should be avoided if possible. Panobinostat is also not recommended in patients with a recent history of myocardial infarction.

Panobinostat toxicity profile necessitated the development of strategies for alleviating drug-induced toxicity. Hematologic toxicities are commonly described since most of the data are extracted from studies where panobinostat is combined to bortezomib and some of them may also be attributed to bortezomib-induced hematologic toxicity. Thrombocytopenia is a frequently reported adverse event (AE) in panobinostat regimens. It is attributed to the inhibition of megakaryocytes and the reduced release of proplatelets. Usually, delay or reduction of panobinostat dose is sufficient for management of thrombocytopenia. Appearance of diarrhea usually requires drug interruption, while in more severe forms (grade 3 or 4), drug dose reduction or even discontinuation may be necessary. Diarrhea can be a debilitating adverse event that may result in dehydration and electrolyte imbalance. Appropriate hydration, restoration of electrolytes, and prompt initiation of appropriate antidiarrheal medication are usually required. Regular electrolyte monitoring and prophylactic antiemetic therapy should also be considered.

Besides bortezomib, as already reported, panobinostat has been combined with other classes of antimyeloma agents. Many common treatment-emergent AEs may be attributed to the overlapping toxicity profiles of the various classes of agents involved. Despite the relatively limited information regarding toxicity profile of the combination of carfilzomib and panobinostat, safety profile of carfilzomibpanobinostat combination, particularly regarding thrombocytopenia, neurotoxicity, fatigue, and gastrointestinal disorders, compares favorably with that of panobinostat and bortezomib [77, 78]. Carfilzomib-panobinostat regimen necessitated less-frequent dose reductions due to toxicity, compared to established bortezomib-panobinostat-dexamethasone regimen. Combination of lenalidomide with panobinostat was also well tolerated with less commonly severe thrombocytopenia, fatigue, and diarrhea, compared to bortezomib, panobinostat, and dexamethasone standard regimen [79]. Regarding patients that received more complex combinations like VRD-panobinostat regimen [84, 85], they developed less frequently severe gastrointestinal toxic effects, at a cost of more often severe hematologic adverse events, particularly thrombocytopenia. In these patients, most frequent hematological grades 1-2 event was anemia and grade 3 event was thrombocytopenia, respectively. Most-frequent grade 3 nonhematological adverse events were fatigue and diarrhea. Despite that $45 \%$ of patients underwent dose reduction, no patient necessitated panobinostat's dose interruption.

\section{Current Therapeutic Landscape in Multiple Myeloma and Future Directions}

HDAC inhibitors represent a novel class of therapeutic agents with a unique mechanism of action. Panobinostat effectiveness as a part of combinatorial regimens granted its approval in the third line of treatment combined with bortezomib and dexamethasone. However, since panobinostat's approval, therapeutic armamentarium for efficient management of refractory/relapsed multiple myeloma patients has tremendously evolved. Second-generation novel agents like carfilzomib and pomalidomide, anti-CD38 monoclonal antibodies, and anti-SLAM7 monoclonal antibodies have proven therapeutic efficacy and have been incorporated in current clinical practice, forming the current landscape for multiple myeloma treatment.

In order to increase therapeutic efficacy, combinations of novel agents like monoclonal antibodies with IMiDs and proteasome inhibitors have been widely explored. These combinations attained deeper responses with prolonged disease control and manageable toxicity profile. Unfortunately, despite recent advances in myeloma management, almost all of patients will eventually relapse, highlighting the unmet medical need for agents with novel mechanisms of actions that will remain active in relapsed/refractory multiple myeloma patients or will resensitize myeloma patients to previously administered therapies.

Panobinostat is an effective drug with a predictable and manageable toxicity profile. Gastrointestinal toxicity in the form of diarrhea, hematologic toxicity, and rare cardiac events in the form of arrhythmias due to QT prolongation should be always taken under concern. Tolerability of this agent could be further increased by careful, proactive adverse event management. Despite reported toxicity, targeting HDAC remains an effective therapeutic option in selected subgroups of refractory/relapsed multiple myeloma patients. Panobinostat has demonstrated in phase II panorama 2 trial [71] the potential to resensitize heavily pretreated patients, refractory to bortezomib, achieving an overall response rate of $34.5 \%$ and a clinical benefit rate of $52.7 \%$.

Moreover, ongoing clinical trials (Panorama 3) have been designed in order to investigate the safety and efficacy of three different regimens of panobinostat $(20 \mathrm{mg}$ TIW, $20 \mathrm{mg}$ BIW, and $10 \mathrm{mg}$ TIW) in combination with bortezomib in subcutaneous form and dexamethasone. This trial is going to identify if reduction in the dosing schedule of panobinostat and subcutaneous administration of bortezomib (which is the current standard procedure) may offer an effective regimen with more tolerable toxicity. Besides 
TABLE 2: Summary of all ongoing active trials investigating panobinostat in patients with MM.

\begin{tabular}{|c|c|c|c|c|c|}
\hline $\mathrm{A} / \mathrm{a}$ & $\begin{array}{l}\text { NCT number } \\
\text { (study name) }\end{array}$ & Phase & Comparing arms & Intervention/treatment & Patients characteristics \\
\hline 1 & NCT01496118 & $\mathrm{I} / \mathrm{II}$ & $\begin{array}{l}\text { Experimental: carfilzomib and } \\
\text { panobinostat }\end{array}$ & Carfilzomib and panobinostat & $\begin{array}{l}\text { Relapse/refractory after at least } \\
\text { one previous bortezomib- } \\
\text { containing regimen }\end{array}$ \\
\hline 2 & NCT01965353 & I & $\begin{array}{l}\text { Experimental: panobinostat, } \\
\text { dexamethasone, lenalidomide, } \\
\text { and bortezomib }\end{array}$ & $\begin{array}{l}\text { Dexamethasone and lenalidomide } \\
\text { and bortezomib and panobinostat }\end{array}$ & $\begin{array}{l}\text { Relapse/refractory after at least } \\
\text { two lines of treatment }\end{array}$ \\
\hline 3 & NCT02722941 & II & $\begin{array}{l}\text { Panobinostat: } 20 \mathrm{mg} \text { by mouth } \\
\text { three [3] times per week, every } \\
\text { other week, of a } 28 \text {-day schedule } \\
\text { Panobinostat: } 10 \mathrm{mg} \text { by mouth } \\
\text { daily for seven [7] days, every } \\
\text { other week, of a } 28 \text {-day schedule }\end{array}$ & $\begin{array}{l}\text { Panobinostat as maintenance } \\
\text { therapy after HDT + ASCT }\end{array}$ & $\begin{array}{l}\text { Patients must have achieved at } \\
\text { least PR after ASCT }\end{array}$ \\
\hline 4 & NCT03256045 & II & $\begin{array}{l}\text { Experimental: carfilzomib and } \\
\text { panobinostat }\end{array}$ & Carfilzomib and panobinostat & $\begin{array}{l}\text { Relapse/refractory after at least } \\
\text { one previous line of treatment }\end{array}$ \\
\hline 5 & NCT01301807 & I & $\begin{array}{l}\text { Experimental: carfilzomib and } \\
\text { panobinostat }\end{array}$ & Carfilzomib and panobinostat & $\begin{array}{c}\text { Relapsed/refractory MM with } \\
\text { failure to at least two lines of MM } \\
\text { treatment including at least one } \\
\text { IMiD (thalidomide, } \\
\text { lenalidomide) and proteasome } \\
\text { inhibitor (bortezomib) }\end{array}$ \\
\hline 6 & NCT02057640 & $\mathrm{I} / \mathrm{II}$ & $\begin{array}{l}\text { Experimental: ixazomib, } \\
\text { panobinostat, and } \\
\text { dexamethasone }\end{array}$ & $\begin{array}{l}\text { Ixazomib and panobinostat and } \\
\text { dexamethasone }\end{array}$ & $\begin{array}{c}\text { Relapsed/refractory MM after } \\
\text { receiving at least one IMiD } \\
\text { (thalidomide, lenalidomide) and } \\
\text { proteasome inhibitor } \\
\text { (bortezomib) }\end{array}$ \\
\hline 7 & NCT00918333 & $\mathrm{I} / \mathrm{II}$ & $\begin{array}{l}\text { Experimental: everolimus and } \\
\text { panobinostat }\end{array}$ & Everolimus and panobinostat & $\begin{array}{l}\text { Relapsed/refractory myeloma } \\
\text { requiring therapy, who have } \\
\text { failed, are unable to tolerate, or } \\
\text { refused other available active } \\
\text { therapies }\end{array}$ \\
\hline 8 & NCT02506959 & II & $\begin{array}{l}\text { Experimental: panobinostat, } \\
\text { gemcitabine hydrochloride, } \\
\text { busulfan, and melphalan ASCT }\end{array}$ & $\begin{array}{c}\text { Panobinostat, Gemcitabine } \\
\text { Hydrochloride, Busulfan, and } \\
\text { melphalan ASCT }\end{array}$ & Refractory/relapsed myeloma \\
\hline 9 & NCT02654990 & II & $\begin{array}{l}\text { Experimental: panobinostat, } \\
\text { bortezomib, and dexamethasone } \\
\text { Investigate } 3 \text { different } \\
\text { panobinostat regimens }\end{array}$ & $\begin{array}{l}\text { Panobinostat and bortezomib and } \\
\text { dexamethasone }\end{array}$ & $\begin{array}{l}\text { Refractory/relapsed myeloma } \\
\text { with } 1 \text { to } 4 \text { prior lines of treatment }\end{array}$ \\
\hline
\end{tabular}

bortezomib, panobinostat has demonstrated in initial phase I/II trials a remarkable synergistic activity with carfilzomib and IMiDs, offering clinical benefit in heavily pretreated, refractory patients. Noteworthy, carfilzomib monotherapy in heavily pretreated (at least 4-5 previous lines of treatment) patients is reported to be far less efficient $[105,106]$ than combined with panobinostat, highlighting a potent clinical synergy. Additional trials should focus on panobinostat's ability to resensitize multiple myeloma patients refractory/ relapsed to second-generation IMIDs, newer proteasome inhibitors, and monoclonal antibodies, besides its ability to augment their therapeutic efficacy [87], as reported with other HDAC inhibitors [107-109]. Moreover, these encouraging results should warrant further confirmation in larger clinical trials. Overall, drug toxicity profile and unique mechanism of action render panobinostat rather a more suitable candidate for multidrug regimens in heavily pretreated patients with aggressive disease behavior than in untreated patients or in first relapse. Ongoing trials (Table 2) are going to shed light on the optimal timing, schedule, and combinations of panobinostat in order to attain the maximum benefit from this class of anticancer agents and optimize treatment tolerability.

\section{Conclusion}

Despite significant advances in the therapeutic management of multiple myeloma, panobinostat remains an important therapeutic option for refractory/relapsed multiple myeloma patients. Available data from multiple clinical trials confirm that it remains an effective therapy even in heavily pretreated patients. HDAC inhibitors like panobinostat, as a part of multidrug combinations, may reform the current multiple myeloma treatment landscape allowing patients to achieve improved quality of response and prolonged survival, with affordable toxicity. 


\section{Conflicts of Interest}

The authors declare that they have no conflicts of interest.

\section{References}

[1] J. A. Katzel, P. Hari, and D. H. Vesole, "Multiple myeloma: charging toward a bright future," CA: A Cancer Journal for Clinicians, vol. 57, no. 5, pp. 301-318, 2007.

[2] R. A. Kyle and S. V. Rajkumar, "Multiple myeloma," New England Journal of Medicine, vol. 351, no. 18, pp. 1860-1873, 2004.

[3] A. Palumbo and K. Anderson, "Multiple myeloma," New England Journal of Medicine, vol. 364, no. 11, pp. 1046-1060, 2011.

[4] https://www.cancer.org/content/dam/cancer-org/research/ cancer-facts-and-statistics/annual-cancer-facts-and-figures/ 2018/cancer-facts-and-figures-2018.pdf.

[5] F. Bray, J. Ferlay, I. Soerjomataram, R. L. Siegel, L. A. Torre, and A. Jemal, "Global cancer statistics 2018: GLOBOCAN estimates of incidence and mortality worldwide for 36 cancers in 185 countries," CA: A Cancer Journal for Clinicians, vol. 68, no. 6, pp. 394-424, 2018.

[6] S. V. Rajkumar, "Updated diagnostic criteria and staging system for multiple myeloma," American Society of Clinical Oncology Educational Book, vol. 35, no. 36, pp. e418-e423, 2016.

[7] E. E. Osgood, "The survival time of patients with plasmocytic myeloma," Cancer Chemotherapy Reports, vol. 9, no. 9, pp. 1-10, 1960.

[8] D. E. Bergsagel, C. C. Sprague, C. Austin, and K. M. Griffith, "Evaluation of new chemotherapeutic agents in the treatment of multiple myeloma. IV. L-Phenylalanine mustard (NSC-8806)," Cancer Chemotherapy Reports, vol. 21, no. 21, pp. 87-99, 1962.

[9] S. Lonial, L. H. Boise, and J. Kaufman, "How I treat high-risk myeloma," Blood, vol. 126, no. 13, pp. 1536-1543, 2015.

[10] M. L. Khan, C. B. Reeder, S. K. Kumar et al., "A comparison of lenalidomide/dexamethasone versus cyclophosphamide/ lenalidomide/dexamethasone versus cyclophosphamide/ bortezomib/dexamethasone in newly diagnosed multiple myeloma," British Journal of Haematology, vol. 156, no. 3, pp. 326-333, 2011.

[11] P. G. Richardson, E. Weller, S. Lonial et al., "Lenalidomide, bortezomib, and dexamethasone combination therapy in patients with newly diagnosed multiple myeloma," Blood, vol. 116, no. 5, pp. 679-686, 2010.

[12] A. K. Stewart, S. V. Rajkumar, M. A. Dimopoulos et al., "Carfilzomib, lenalidomide, and dexamethasone for relapsed multiple myeloma," The New England Journal of Medicine, vol. 372, no. 2, pp. 142-152, 2014.

[13] J. S. Miguel, K. Weisel, P. Moreau et al., "Pomalidomide plus low-dose dexamethasone versus high-dose dexamethasone alone for patients with relapsed and refractory multiple myeloma (MM-003): a randomised, open-label, phase 3 trial," The Lancet Oncology, vol. 14, no. 11, pp. 1055-1066, 2013.

[14] T. McElwain and R. L. Powles, "High-dose intravenous melphalan for plasma-cell leukaemia and myeloma," The Lancet, vol. 322, no. 8354, pp. 822-824, 1983.

[15] M. Attal, J.-L. Harousseau, A.-M. Stoppa et al., "A prospective, randomized trial of autologous bone marrow transplantation and chemotherapy in multiple myeloma,"
New England Journal of Medicine, vol. 335, no. 2, pp. 91-97, 1996.

[16] J. A. Child, G. J. Morgan, F. E. Davies et al., "High-dose chemotherapy with hematopoietic stem-cell rescue for multiple myeloma," New England Journal of Medicine, vol. 348, no. 19, pp. 1875-1883, 2003.

[17] M. A. Dimopoulos, P. Moreau, A. Palumbo et al., "Carfilzomib and dexamethasone versus bortezomib and dexamethasone for patients with relapsed or refractory multiple myeloma (ENDEAVOR): a randomised, phase 3, open-label, multicentre study," The Lancet Oncology, vol. 17, no. 1, pp. 27-38, 2016.

[18] S. M. Hoy, "Pomalidomide: a review in relapsed and refractory multiple myeloma," Drugs, vol. 77, no. 17, pp. 1897-1908, 2017.

[19] Y. Zou, X. Ma, H. Yu et al., "Carfilzomib/pomalidomide single-agent or in combination with other agents for the management of relapsed/refractory multiple myeloma: a meta-analysis of 37 trials," Oncotarget, vol. 8, no. 24, pp. 39805-39817, 2017.

[20] A. Chari, A. Suvannasankha, J. W. Fay et al., "Daratumumab plus pomalidomide and dexamethasone in relapsed and/or refractory multiple myeloma," Blood, vol. 130, no. 8, pp. 974-981, 2017.

[21] M. A. Dimopoulos, A. Oriol, H. Nahi et al., "Daratumumab, lenalidomide, and dexamethasone for multiple myeloma," New England Journal of Medicine, vol. 375, no. 14, pp. 1319-1331, 2016.

[22] A. Palumbo, A. Chanan-Khan, K. Weisel et al., "Daratumumab, bortezomib, and dexamethasone for multiple myeloma," New England Journal of Medicine, vol. 375, no. 8, pp. 754-766, 2016.

[23] M.-V. Mateos, M. A. Dimopoulos, M. Cavo et al., "Daratumumab plus bortezomib, melphalan, and prednisone for untreated myeloma," New England Journal of Medicine, vol. 378 , no. 6 , pp. 518-528, 2018.

[24] H. M. Lokhorst, T. Plesner, J. P. Laubach et al., "Targeting CD38 with daratumumab monotherapy in multiple myeloma," New England Journal of Medicine, vol. 373, no. 13, pp. 1207-1219, 2015.

[25] T. Martin, R. Baz, D. M. Benson et al., "A phase 1b study of isatuximab plus lenalidomide and dexamethasone for relapsed/refractory multiple myeloma," Blood, vol. 129, no. 25, pp. 3294-3303, 2017.

[26] N. W. C. J. van de Donk, P. G. Richardson, and F. Malavasi, "CD38 antibodies in multiple myeloma: back to the future," Blood, vol. 131, no. 1, pp. 13-29, 2018.

[27] P. G. Richardson, M. Attal, F. Campana et al., "Isatuximab plus pomalidomide/dexamethasone versus pomalidomide/ dexamethasone in relapsed/refractory multiple myeloma: ICARIA phase III study design," Future Oncology, vol. 14, no. 11, pp. 1035-1047, 2018.

[28] T. Martin, S. Strickland, M. Glenn et al., "Phase I trial of isatuximab monotherapy in the treatment of refractory multiple myeloma," Blood Cancer Journal, vol. 9, no. 4, p. 41, 2019.

[29] S. Lonial, M. Dimopoulos, A. Palumbo et al., "Elotuzumab therapy for relapsed or refractory multiple myeloma. Elotuzumab therapy for relapsed or refractory multiple myeloma," The New England Journal of Medicine, vol. 373, no. 7, pp. 621-631, 2015.

[30] M. A. Dimopoulos, D. Dytfeld, S. Grosicki et al., "Elotuzumab plus pomalidomide and dexamethasone for multiple 
myeloma," New England Journal of Medicine, vol. 379, no. 19, pp. 1811-1822, 2018.

[31] A. Jakubowiak, M. Offidani, B. Pégourie et al., "Randomized phase 2 study: elotuzumab plus bortezomib/dexamethasone vs. bortezomib/dexamethasone for relapsed/refractory MM," Blood, vol. 127, no. 23, pp. 2833-2840, 2016.

[32] S. K. Kumar, J. H. Lee, J. J. Lahuerta et al., "Risk of progression and survival in multiple myeloma relapsing after therapy with IMiDs and bortezomib: a multicenter international myeloma working group study," Leukemia, vol. 26, no. 1, pp. 149-157, 2012.

[33] D. Dingli, S. Ailawadhi, P. L. Bergsagel et al., "Therapy for relapsed multiple myeloma," Mayo Clinic Proceedings, vol. 92, no. 4, pp. 578-598, 2017.

[34] J. B. Egan, C.-X. Shi, W. Tembe et al., "Whole-genome sequencing of multiple myeloma from diagnosis to plasma cell leukemia reveals genomic initiating events, evolution, and clonal tides," Blood, vol. 120, no. 5, pp. 1060-1066, 2012.

[35] G. J. Morgan, B. A. Walker, and F. E. Davies, "The genetic architecture of multiple myeloma," Nature Reviews Cancer, vol. 12 , no. 5, pp. 335-348, 2012.

[36] P. Atadja, "Development of the pan-DAC inhibitor panobinostat (LBH589): successes and challenges," Cancer Letters, vol. 280, no. 2, pp. 233-241, 2009.

[37] US Food and Drug Administration, "FDA approves Farydak for treatment of multiple myeloma," 2015, http://www.fda. gov/NewsEvents/Newsroom/PressAnnouncements/ ucm435296.htm.

[38] Novartis, Farydak (Panobinostat) Capsules [Prescribing Information], Novartis, East Hanover, NJ, USA, 2015.

[39] K. Tzogani, P. Hennik, I. Walsh et al., "The European Medicines agency review of panobinostat (Farydak) for the treatment of adult patients with relapsed and/or refractory multiple myeloma," The Oncologist, vol. 22, pp. 1-6, 2017.

[40] C. J. Hall, S. Doss, N. Hay, F. Sutcliffe, and A. Stevens, "NICE guidance on panobinostat for patients with multiple myeloma after at least two previous treatments," The Lancet Oncology, vol. 17, no. 3, pp. 279-280, 2016.

[41] P. D. Gregory, K. Wagner, and W. Hörz, "Histone acetylation and chromatin remodeling," Experimental Cell Research, vol. 265, no. 2, pp. 195-202, 2001.

[42] A. A. Lane and B. A. Chabner, "Histone deacetylase inhibitors in cancer therapy," Journal of Clinical Oncology, vol. 27, no. 32, pp. 5459-5468, 2009.

[43] D. Y. Lee, J. J. Hayes, D. Pruss, and A. P. Wolffe, “A positive role for histone acetylation in transcription factor access to nucleosomal DNA," Cell, vol. 72, no. 1, pp. 73-84, 1993.

[44] P. A. Wolffe, R. A. Rifkind, V. M. Richon, R. Breslow, T. Miller, and W. K. Kelly, "Histone deacetylases and cancer: causes and therapies," Nature Reviews Cancer, vol. 1, no. 3, pp. 194-202, 2001.

[45] S. Ropero and M. Esteller, "The role of histone deacetylases (HDACs) in human cancer," Molecular Oncology, vol. 1, no. 1, pp. 19-25, 2007.

[46] M. F. Kaiser, D. C. Johnson, P. Wu et al., "Global methylation analysis identifies prognostically important epigenetically inactivated tumor suppressor genes in multiple myeloma," Blood, vol. 122, no. 2, pp. 219-226, 2013.

[47] J. Moreaux, T. Reme, W. Leonard et al., "Gene expressionbased prediction of myeloma cell sensitivity to histone deacetylase inhibitors," British Journal of Cancer, vol. 109, no. 3, pp. 676-685, 2013.

[48] S. Mithraprabhu, A. Kalff, A. Chow, T. Khong, and A. Spencer, "Dysregulated class I histone deacetylases are indicators of poor prognosis in multiple myeloma," Epigenetics, vol. 9, no. 11, pp. 1511-1520, 2014.

[49] D. S. Siegel, M. Dimopoulos, S. Jagannath et al., "VANTAGE 095: an international, multicenter, open-label study of vorinostat (MK-0683) in combination with bortezomib in patients with relapsed and refractory multiple myeloma," Clinical Lymphoma Myeloma and Leukemia, vol. 16, no. 6, pp. 329-334e1, 2016.

[50] P. M. Voorhees, C. Gasparetto, D. T. Moore, D. Winans, R. Z. Orlowski, and D. D. Hurd, "Final results of a phase 1 study of vorinostat, pegylated liposomal doxorubicin, and bortezomib in relapsed or refractory multiple myeloma," Clinical Lymphoma Myeloma and Leukemia, vol. 17, no. 7, pp. 424-432, 2017.

[51] D. W. Sborov, D. M. Benson, N. Williams et al., "Lenalidomide and vorinostat maintenance after autologous transplant in multiple myeloma," British Journal of Haematology, vol. 171, no. 1, pp. 74-83, 2015.

[52] D. T. Vogl, N. Raje, S. Jagannath et al., "Ricolinostat, the first selective histone deacetylase 6 inhibitor, in combination with bortezomib and dexamethasone for relapsed or refractory multiple myeloma," Clinical Cancer Research, vol. 23, no. 13, pp. 3307-3315, 2017.

[53] A. J. Yee, W. I. Bensinger, J. G. Supko et al., "Ricolinostat plus lenalidomide, and dexamethasone in relapsed or refractory multiple myeloma: a multicentre phase $1 \mathrm{~b}$ trial," The Lancet Oncology, vol. 17, no. 11, pp. 1569-1578, 2016.

[54] R. Niesvizky, S. Ely, T. Mark et al., "Phase 2 trial of the histone deacetylase inhibitor romidepsin for the treatment of refractory multiple myeloma," Cancer, vol. 117, no. 2, pp. 336-342, 2011.

[55] https://clinicaltrials.gov study identifier NCT02400242.

[56] Y. Kawaguchi, J. J. Kovacs, A. McLaurin, J. M. Vance, A. Ito, and T.-P. Yao, "The deacetylase HDAC6 regulates aggresome formation and cell viability in response to misfolded protein stress," Cell, vol. 115, no. 6, pp. 727-738, 2003.

[57] L. Catley, E. Weisberg, T. Kiziltepe et al., "Aggresome induction by proteasome inhibitor bortezomib and $\alpha$-tubulin hyperacetylation by tubulin deacetylase (TDAC) inhibitor LBH589 are synergistic in myeloma cells," Blood, vol. 108, no. 10, pp. 3441-3449, 2006.

[58] J. Kikuchi, T. Wada, R. Shimizu et al., "Histone deacetylases are critical targets of bortezomib-induced cytotoxicity in multiple myeloma," Blood, vol. 116, no. 3, pp. 406-417, 2010.

[59] E. M. Ocio, D. Vilanova, P. Atadja et al., "In vitro and in vivo rationale for the triple combination of panobinostat (LBH589) and dexamethasone with either bortezomib or lenalidomide in multiple myeloma," Haematologica, vol. 95, no. 5, pp. 794-803, 2010.

[60] T. Hideshima, P. G. Richardson, and K. C. Anderson, "Mechanism of action of proteasome inhibitors and deacetylase inhibitors and the biological basis of synergy in multiple myeloma," Molecular Cancer Therapeutics, vol. 10, no. 11, pp. 2034-2042, 2011.

[61] S. Clive, M. M. Woo, T. Nydam, L. Kelly, M. Squier, and M. Kagan, "Characterizing the disposition, metabolism, and excretion of an orally active pan-deacetylase inhibitor, panobinostat, via trace radiolabeled $14 \mathrm{C}$ material in advanced cancer patients," Cancer Chemotherapy and Pharmacology, vol. 70, no. 4, pp. 513-522, 2012.

[62] M. Van Veggel, E. Westerman, and P. Hamberg, "Clinical pharmacokinetics and pharmacodynamics of panobinostat," Clinical Pharmacokinetics, vol. 57, no. 1, pp. 21-29, 2018. 
[63] N. R. Srinivas, "Clinical pharmacokinetics of panobinostat, a novel histone deacetylase (HDAC) inhibitor: review and perspectives," Xenobiotica; the Fate of Foreign Compounds in Biological Systems, vol. 47, no. 47, pp. 354-368, 2017.

[64] M. Savelieva, M. M. Woo, H. Schran, S. Mu, J. Nedelman, and R. Capdeville, "Population pharmacokinetics of intravenous and oral panobinostat in patients with hematologic and solid tumors," European Journal of Clinical Pharmacology, vol. 71, no. 6, pp. 663-672, 2015.

[65] S. Sharma, P. O. Witteveen, M. P. Lolkema et al., "A phase I, open-label, multicenter study to evaluate the pharmacokinetics and safety of oral panobinostat in patients with advanced solid tumors and varying degrees of renal function," Cancer Chemotherapy and Pharmacology, vol. 75, no. 1, pp. 87-95, 2015.

[66] M. Slingerland, D. Hess, S. Clive et al., "A phase I, open-label, multicenter study to evaluate the pharmacokinetics and safety of oral panobinostat in patients with advanced solid tumors and various degrees of hepatic function," Cancer Chemotherapy and Pharmacology, vol. 74, no. 5, pp. 10891098, 2014.

[67] K. Wahaib, A. E. Beggs, H. Campbell, L. Kodali, and P. D. Ford, "Panobinostat: a histone deacetylase inhibitor for the treatment of relapsed or refractory multiple myeloma," American Journal of Health-System Pharmacy, vol. 73, no. 7, pp. 441-450, 2016.

[68] D. J. DeAngelo, A. Spencer, K. N. Bhalla et al., "Phase Ia/II, two-arm, open-label, dose-escalation study of oral panobinostat administered via two dosing schedules in patients with advanced hematologic malignancies," Leukemia, vol. 27, no. 8, pp. 1628-1636, 2013.

[69] J. L. Wolf, D. Siegel, H. Goldschmidt et al., "Phase II trial of the pan-deacetylase inhibitor panobinostat as a single agent in advanced relapsed/refractory multiple myeloma," Leukemia \& Lymphoma, vol. 53, no. 9, pp. 1820-1823, 2012.

[70] J. F. San-Miguel, P. G. Richardson, A. Günther et al., "Phase Ib study of panobinostat and bortezomib in relapsed or relapsed and refractory multiple myeloma," Journal of Clinical Oncology, vol. 31, no. 29, pp. 3696-3703, 2013.

[71] P. G. Richardson, R. L. Schlossman, M. Alsina et al., "PANORAMA 2: panobinostat in combination with bortezomib and dexamethasone in patients with relapsed and bortezomib-refractory myeloma," Blood, vol. 122, no. 14, pp. 2331-2337, 2013.

[72] J. F. San-Miguel, V. T. Hungria, S. S. Yoon et al., "Panobinostat plus bortezomib and dexamethasone versus placebo plus bortezomib and dexamethasone in patients with relapsed or relapsed and refractory multiple myeloma: a multicentre, randomized, double-blind phase 3 trial," The Lancet Oncology, vol. 15, no. 11, pp. 1195-1206, 2014.

[73] J. F. San-Miguel, V. T. M. Hungria, S.-S. Yoon et al., "Overall survival of patients with relapsed multiple myeloma treated with panobinostat or placebo plus bortezomib and dexamethasone (the PANORAMA 1 trial): a randomised, placebo-controlled, phase 3 trial," The Lancet Haematology, vol. 3, no. 11, pp. e506-e515, 2016.

[74] S. Mangiacavalli, L. Pochintesta, E. Ravelli et al., "Panobinostat in combination with bortezomib and dexamethasone as induction therapy in patients with multiple myeloma, candidates for autologous transplant," Leukemia \& Lymphoma, vol. 56, no. 6, pp. 1901-1902, 2015.

[75] V. L. Hansen, M. Coleman, S. Elkins et al., "An expanded treatment protocol of panobinostat plus bortezomib and dexamethasone in patients with previously treated myeloma," Clinical Lymphoma Myeloma and Leukemia, vol. 18, no. 6, pp. 400-407, 2018.

[76] L. Gao, M. Gao, G. Yang et al., "Synergistic activity of carfilzomib and panobinostat in multiple myeloma cells via modulation of ROS generation and ERK1/2," BioMed Research International, vol. 2015, no. 9, Article ID 459052, 2015.

[77] J. G. Berdeja, L. L. Hart, J. R. Mace et al., "Phase I/II study of the combination of panobinostat and carfilzomib in patients with relapsed/refractory multiple myeloma," Haematologica, vol. 100, no. 5, pp. 670-676, 2015.

[78] J. L. Kaufman, R. Mina, A. J. Jakubowiak et al., “Combining carfilzomib and panobinostat to treat relapsed/refractory multiple myeloma: results of a multiple myeloma research consortium phase i study," Blood Cancer Journal, vol. 9, no. 1, p. 3, 2019.

[79] A. Chari, H. J. Cho, A. Dhadwal et al., "A phase 2 study of panobinostat with lenalidomide and weekly dexamethasone in myeloma," Blood Advances, vol. 1, no. 19, pp. 1575-1583, 2017.

[80] R. Rosato, S. Hock, P. Dent, Y. Dai, and S. Dai, "LBH-589 (panobinostat) potentiates fludarabine anti-leukemic activity through a JNK- and XIAP-dependent mechanism," Leukemia Research, vol. 36, no. 4, pp. 491-498, 2012.

[81] E. Sanchez, J. Shen, J. Steinberg et al., "The histone deacetylase inhibitor LBH589 enhances the anti-myeloma effects of chemotherapy in vitro and in vivo," Leukemia Research, vol. 35, no. 3, pp. 373-379, 2011.

[82] M. Offidani, L. Corvatta, A. M. Liberati, S. Pulini, S. Ballanti, and S. Bringhen, "Updated results of a phase 2 study of panobinostat combined with melphalan, thalidomide and prednisone (MPT) in relapsed/refractory multiple myeloma," Leukemia \& Lymphoma, vol. 59, no. 5, pp. 1271-1273, 2018.

[83] R. Popat, S. R. Brown, L. Flanagan et al., "Bortezomib, thalidomide, dexamethasone, and panobinostat for patients with relapsed multiple myeloma (MUK-six): a multicentre, open-label, phase 1/2 trial," The Lancet Haematology, vol. 3, no. 12, pp. e572-e580, 2016.

[84] E. E. Manasanch, J. J. Shah, H. C. Lee et al., "Bortezomib, lenalidomide, and dexamethasone with panobinostat for front-line treatment of patients with multiple myeloma who are eligible for transplantation: a phase 1 trial," The Lancet Haematology, vol. 5, no. 12, pp. e628-e640, 2018.

[85] J. Laubach, S. A. Tuchman, J. Rosenblatt et al., "Phase 1 b study of panobinostat in combination with lenalidomide, bortezomib, and dexamethasone in relapsed refractory multiple myeloma," Journal of Clinical Oncology, vol. 34, no. 15_suppl, p. 8014, 2016.

[86] A. M. Rajan, F. K. Buadi, V. Rajkumar et al., "Effective use of panobinostat in combination with other active agents in myeloma in a novel five-drug combination: case report and interesting observations," American Journal of Hematology, vol. 91, no. 2, pp. E5-E6, 2016.

[87] E. García-Guerrero, T. Gogishvili, S. Danhof et al., "Panobinostat induces CD38 upregulation and augments the antimyeloma efficacy of daratumumab," Blood, vol. 129, no. 25 , pp. 3386-3388, 2017.

[88] I. S. Nijhof, T. Casneuf, J. van Velzen et al., "CD38 expression and complement inhibitors affect response and resistance to daratumumab therapy in myeloma," Blood, vol. 128 , no. 7 , pp. 959-970, 2016.

[89] T. Jelinek, B. Paiva, and R. Hajek, "Update on PD-1/PD-L1 inhibitors in multiple myeloma," Frontiers in Immunology, vol. 9, p. 2431, 2018. 
[90] F. Costa, R. Das, J. Kini Bailur et al., "Checkpoint inhibition in myeloma: opportunities and challenges," Frontiers in Immunology, vol. 9, p. 2204, 2018.

[91] A. Badros, E. Hyjek, N. Ma et al., "Pembrolizumab, pomalidomide, and low-dose dexamethasone for relapsed/ refractory multiple myeloma," Blood, vol. 130, no. 10, pp. 1189-1197, 2017.

[92] M. V. Mateos, H. Blacklock, F. Schjesvold et al., "Pembrolizumab plus pomalidomide and dexamethasone for patients with relapsed or refractory multiple myeloma (KEYNOTE-183): a randomised, open-label, phase 3 trial," The Lancet Haematology, vol. 6, no. 6, pp. e459-e469, 2019.

[93] D. M. Woods, A. L. Sodre, A. Villagra, A. Sarnaik, E. M. Sotomayor, and J. Weber, "HDAC inhibition upregulates PD-1 ligands in melanoma and augments immunotherapy with pd-1 blockade," Cancer Immunology Research, vol. 3, no. 12, pp. 1375-1385, 2015.

[94] L. Booth, J. L. Roberts, A. Poklepovic et al., "HDAC inhibitors enhance the immunotherapy response of melanoma cells," Oncotarget, vol. 8, no. 47, pp. 83155-83170, 2017.

[95] A. Ray, D. S. Das, Y. Song, D. Chauhan, and K. C. Anderson, "Combination of anti-PD-L1 antibody with IMiD immunomodulatory drugs, HDAC inhibitor ACY-1215, bortezomib, or toll-like receptor 9 agonist enhances anti-tumor immunity and cytotoxicity in multiple myeloma," Blood, vol. 126, no. 23, p. 3014, 2015.

[96] A. Ray, D. S. Das, Y. Song, T. Hideshima, D. Chauhan, and K. C. Anderson, "Combination of a novel HDAC 6 inhibitor ACY-241 with anti-PD-L1 antibody enhances anti-tumor immunity and cytotoxicity in multiple myeloma," Blood, vol. 128 , no. 22, p. 382, 2016.

[97] M. J. Bishton, S. J. Harrison, B. P. Martin et al., "Deciphering the molecular and biologic processes that mediate histone deacetylase inhibitor-induced thrombocytopenia," Blood, vol. 117, no. 13, pp. 3658-3668, 2011.

[98] S. Lonial, E. K. Waller, P. G. Richardson et al., "Risk factors and kinetics of thrombocytopenia associated with bortezomib for relapsed, refractory multiple myeloma," Blood, vol. 106, no. 12, pp. 3777-3784, 2005.

[99] J. F. San-Miguel, V. T. M. Hungria, S.-S. Yoon et al., "Panobinostat plus bortezomib and dexamethasone: impact of dose intensity and administration frequency on safety in the PANORAMA 1 trial," British Journal of Haematology, vol. 179, no. 1, pp. 66-74, 2017.

[100] D. C. Moore, J. R. Arnall, and R. D. Harvey, "Incidence and management of adverse events associated with panobinostat in the treatment of relapsed/refractory multiple myeloma," Journal of Oncology Pharmacy Practice, vol. 25, no. 3, pp. 613-622, 2019.

[101] J. D. Cavenagh and R. Popat, "Optimal management of histone deacetylase inhibitor-related adverse events in patients with multiple myeloma: a focus on panobinostat," Clinical Lymphoma Myeloma and Leukemia, vol. 18, no. 8, pp. 501-507, 2018.

[102] G. Cengiz Seval and M. Beksac, "A comparative safety review of histone deacetylase inhibitors for the treatment of myeloma," Expert Opinion on Drug Safety, vol. 18, no. 7, pp. 563-571, 2019.

[103] F. Giles, T. Fischer, J. Cortes et al., "A phase I study of intravenous LBH589, a novel cinnamic hydroxamic acid analogue histone deacetylase inhibitor, in patients with refractory hematologic malignancies," Clinical Cancer Research, vol. 12, no. 15, pp. 4628-4635, 2006.
[104] L. Zhang, D. Lebwohl, E. Masson, G. Laird, M. R. Cooper, and H. M. Prince, "Clinically relevant QTc prolongation is not associated with current dose schedules of LBH589 (panobinostat)," Journal of Clinical Oncology, vol. 26, no. 2, pp. 332-333, 2008.

[105] S. Iida, T. Watanabe, M. Matsumoto et al., "Carfilzomib monotherapy in Japanese patients with relapsed or refractory multiple myeloma: a phase $1 / 2$ study," Cancer Science, vol. 110, no. 9, pp. 2924-2932, 2019.

[106] R. Hájek, T. Masszi, M. T. Petrucci et al., “A randomized phase III study of carfilzomib vs. low-dose corticosteroids with optional cyclophosphamide in relapsed and refractory multiple myeloma (FOCUS)," Leukemia, vol. 31, no. 1, pp. 107-114, 2017.

[107] T. Hideshima, J. Qi, R. M. Paranal et al., "Discovery of selective small-molecule HDAC6 inhibitor for overcoming proteasome inhibitor resistance in multiple myeloma," Proceedings of the National Academy of Sciences, vol. 113, no. 46, pp. 13162-13167, 2016.

[108] A. Canella, H. Cordero Nieves, D. W. Sborov et al., "HDAC inhibitor AR-42 decreases CD44 expression and sensitizes myeloma cells to lenalidomide," Oncotarget, vol. 6, no. 31, pp. 31134-31150, 2015.

[109] X. Sun, Y. Xie, X. Sun et al., "The selective HDAC6 inhibitor Nexturastat A induces apoptosis, overcomes drug resistance and inhibits tumor growth in multiple myeloma," Bioscience Reports, vol. 39, no. 3, 2019. 


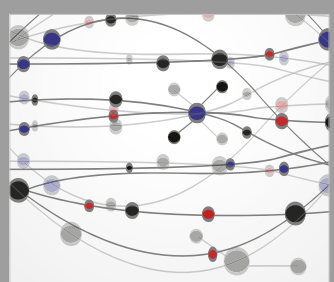

The Scientific World Journal
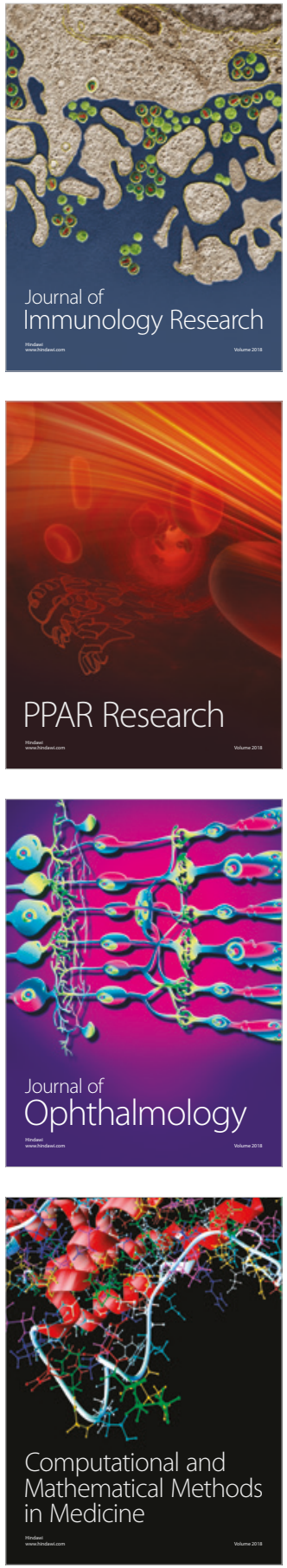

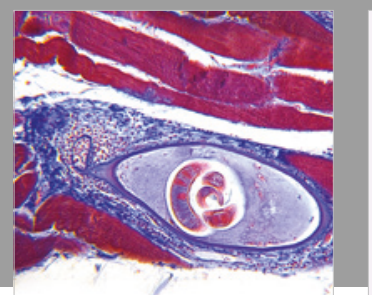

Gastroenterology Research and Practice

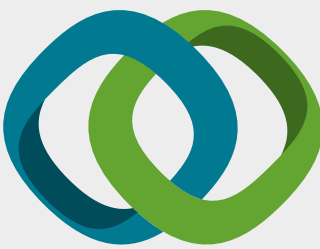

\section{Hindawi}

Submit your manuscripts at

www.hindawi.com
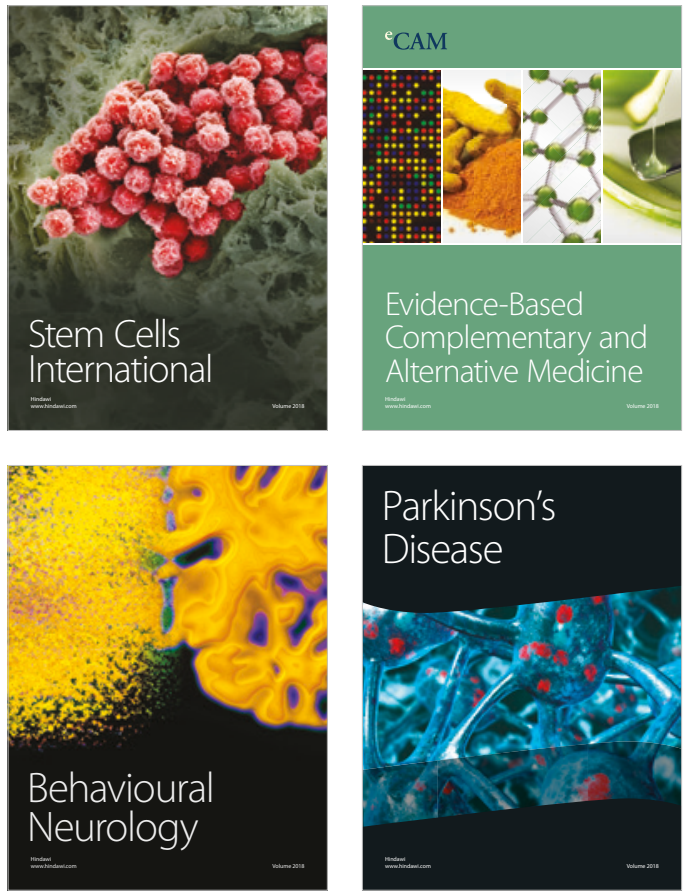

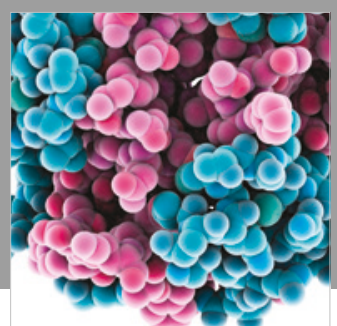

ournal of

Diabetes Research

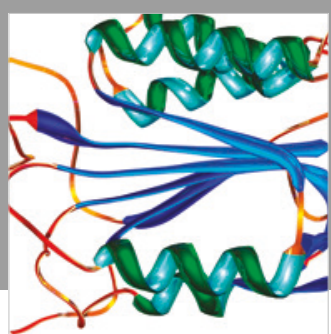

Disease Markers
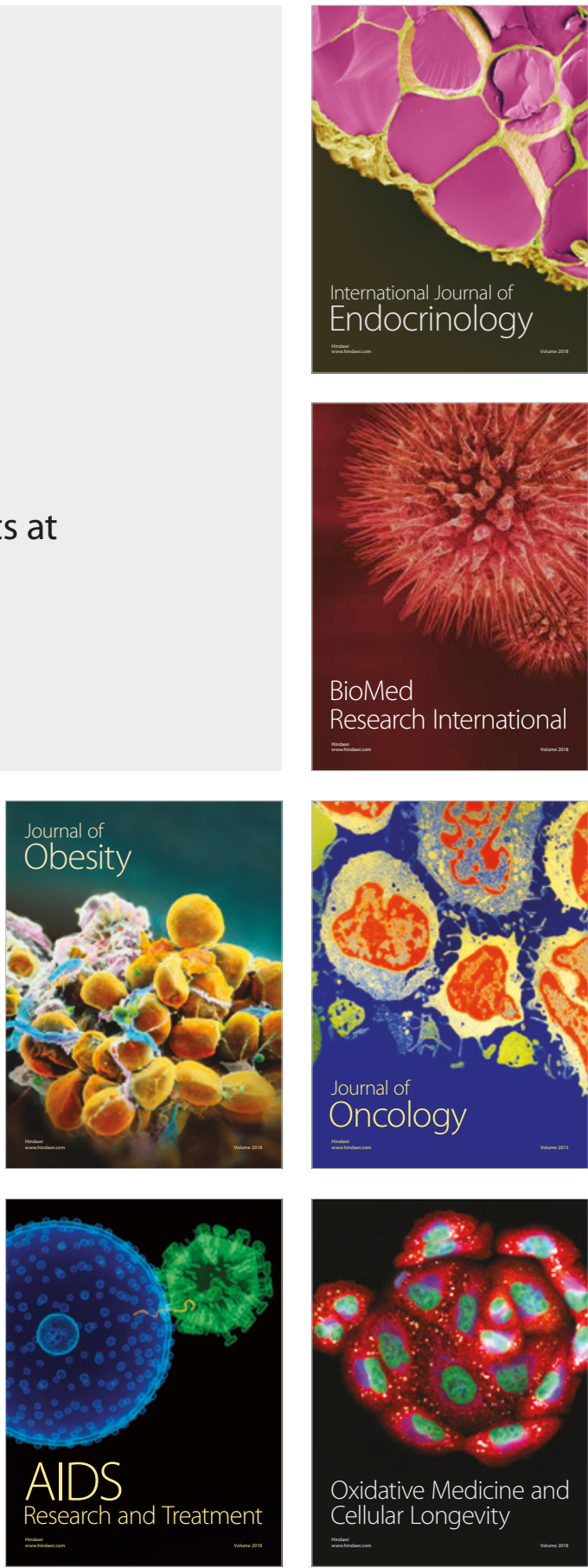